EPJ manuscript No.

(will be inserted by the editor)

\title{
Quantum Brownian motion and the second law of thermodynamics
}

\author{
ILki Kim ${ }^{1 \mathrm{a}}$ and Günter Mahler ${ }^{2}$ \\ ' 1 Department of Physics, North Carolina Central University, Durham, NC 27707, U.S.A. \\ ${ }^{2}$ Institute of Theoretical Physics I, University of Stuttgart, Pfaffenwaldring 57/IV, 70550 Stuttgart, Germany
}

August 17, 2018

\begin{abstract}
We consider a single harmonic oscillator coupled to a bath at zero temperature. As is well known, the oscillator then has a higher average energy than that given by its ground state. Here we show analytically that for a damping model with arbitrarily discrete distribution of bath modes and damping models with continuous distributions of bath modes with cut-off frequencies, this excess energy is less than the work needed to couple the system to the bath, therefore, the quantum second law is not violated. On the other hand, the second law may be violated for bath modes without cut-off frequencies, which are, however, physically unrealistic models.
\end{abstract}

PACS. 03.65.Ud Entanglement and quantum nonlocality - 05.40.-a Fluctuation phenomena, random processes, noise, and Brownian motion - 05.70.-a Thermodynamics

\section{Introduction}

Thermodynamics originally developed as a purely phenomenological description of the effects caused by changes in temperature, pressure, and volume on physical systems at the macroscopic scale. At the heart of thermodynamics there are four well-known laws [1] the zeroth law allows us to define temperature scales and thermometers while the first law is nothing else than a generalized expression of the law of energy conservation. The second law introduces the concept of thermodynamic entropy, which never decreases for an isolated system. The third law states that as a system approaches the zero temperature, the entropy of the system approaches zero. Later on, Boltzmann and his followers created and developed statistical thermodynamics by reducing the phenomenologically described thermodynamics entirely to the scheme of classical statistical mechanics. When quantum mechanics appeared, the statistical thermodynamics had to take into account additional factors offered by quantum mechanics, but the overall structure of thermodynamics, its fundamental laws, and its meaning fit for macroscopic systems remained unchanged since quantum mechanics was believed to play no roles at the macroscopic scale.

A big challenge for thermodynamics arose with the miniaturization of a system under consideration 22; in contrast to common quantum statistical mechanics which is intrinsically based on a vanishingly small coupling between system and bath, the finite coupling strength between them causes some subtleties that must be recog-

\footnotetext{
a e-mail: hannibal.ikim@gmail.com
}

nized. Recent advances in technology have enabled us to experimentally study mesoscopic systems and test various fundamental concepts. The field of nano electro-mechanical systems (NEMS) especially has emerged with a great potential, e.g., in quantum limit detection and amplification [3,4], and welcher-Weg ('which-path') interferometry [5]. Here, the effects of dissipative environments that are negligible in macroscopic resonators become detrimental, and the noise is, therefore, a major limiting factor in control of NEMS resonators. Theoretically, NEMS resonators can be modeled as the simplest form in the scheme of quantum Brownian motion (see [6] for fundamental aspects of quantum Brownian motion). Such a development in various fields related to the quantum statistical and mesoscopic physics has led to considerable interest in the area of quantum and mesoscopic thermodynamics, especially with the question raised on the validity of the thermodynamic laws. Discussions about what is the meaning of quantum thermodynamics [2,7] have started and continued up to now.

The validity of the second law was questioned in the scheme of quantum Brownian motion [2], motivated from the observation of the fact that a single harmonic oscillator coupled to a bath at zero temperature has indeed a higher average energy value than the uncoupled harmonic oscillator ground state (see also [8]), which could not be in accordance with the second law in its Kelvin-Planck form [1] that a system operating in contact with a thermal reservoir cannot produce positive work in its surroundings ( $c f$. for a discussion on the validity of the quantum third law in the low temperature limit, see, e.g., Refs. [9], 
[10]). However, this argument has been shown to be wrong by Ford and O'Connell [11; by means of the generalized Langevin equation they showed, for the well-known Drude model for the spectral density of bath modes, that the apparent excess energy in the coupled harmonic oscillator, however, cannot be used to extract useful work since the minimum value of the work to couple the free oscillator to a bath takes above and beyond this excess energy, therefore, the second law of thermodynamics is inviolate even in the quantum regime (i.e., for cases with non-negligible coupling strengths at temperature $T=0$ without thermal fluctuation). Unfortunately they were unable to explicitly connect their result with its model-independent, deep quantum origin, thus the validity of the quantum second law for a more general form of the spectral density of bath modes, $J(\omega)$ would still remain an open question; actually, in the experimental study of mesoscopic systems one might be able to manipulate the spectral density $J(\omega)$, to some extent, in his own way. In this paper, we would like to discuss the second law for various damping models. We will first show the validity of the second law for a discrete distribution of bath modes by exactly proving the second-law inequality in a simple form obtained from the general treatment of the susceptibility (see Sec. 3). Subsequently, the inequality will be appropriately applied for various continuous distributions of bath modes. It is then found that for damping models with cut-off frequencies, the second law holds, whereas interestingly, we may have its violation for damping models with cut-off frequencyfree $J(\omega)$, which are, however, physically unrealistic (see Sec. (4). Let us begin with a brief review on the basics of the quantum Brownian motion. We will below adopt the notations used in [12].

\section{Basics and its general treatment}

The quantum Brownian motion in consideration is described by the model Hamiltonian

$$
\hat{H}=\hat{H}_{s}+\hat{H}_{b}+\hat{H}_{s b},
$$

where

$$
\begin{aligned}
\hat{H}_{s} & =\frac{\hat{p}^{2}}{2 M}+\frac{M}{2} \omega_{0}^{2} \hat{q}^{2} ; \hat{H}_{b}=\sum_{j=1}^{N}\left(\frac{\hat{p}_{j}^{2}}{2 m_{j}}+\frac{m_{j}}{2} \omega_{j}^{2} \hat{x}_{j}^{2}\right) \\
\hat{H}_{s b} & =-\hat{q} \sum_{j=1}^{N} c_{j} \hat{x}_{j}+\hat{q}^{2} \sum_{j=1}^{N} \frac{c_{j}^{2}}{2 m_{j} \omega_{j}^{2}} .
\end{aligned}
$$

Here, from the hermiticity of Hamiltonian, the coupling constants $c_{j}$ are obviously real-valued. Without any loss of generality, we assume that

$$
\omega_{1} \leq \omega_{2} \leq \cdots \leq \omega_{N-1} \leq \omega_{N} .
$$

By means of the Heisenberg equation of motion for $\hat{p}$ we can derive the quantum Langevin equation

$$
M \ddot{\hat{q}}+M \int_{0}^{t} d s \gamma(t-s) \dot{\hat{q}}(s)+M \omega_{0}^{2} \hat{q}=\hat{\xi}(t),
$$

where we used $\hat{p}=M \dot{\hat{q}}$, and the damping kernel and the noise operator are respectively given by

$$
\begin{aligned}
\gamma(t)= & \frac{1}{M} \sum_{j=1}^{N} \frac{c_{j}^{2}}{m_{j} \omega_{j}^{2}} \cos \left(\omega_{j} t\right) ; \hat{\xi}(t)=-M \gamma(t) \hat{q}(0)+ \\
& \sum_{j=1}^{N} c_{j}\left\{\hat{x}_{j}(0) \cos \left(\omega_{j} t\right)+\frac{\hat{p}_{j}(0)}{m_{j} \omega_{j}} \sin \left(\omega_{j} t\right)\right\} .
\end{aligned}
$$

Introducing the spectral density of bath modes as a characteristic of the bath,

$$
J(\omega)=\pi \sum_{j=1}^{N} \frac{c_{j}^{2}}{2 m_{j} \omega_{j}} \delta\left(\omega-\omega_{j}\right),
$$

we can express the damping kernel as

$$
\gamma(t)=\frac{2}{M} \int_{0}^{\infty} \frac{d \omega}{\pi} \frac{J(\omega)}{\omega} \cos (\omega t) .
$$

Let us apply the Laplace transform to eq. (4) with the aid of 13,14

$$
\begin{aligned}
\mathcal{L}\left\{\cos \left(\omega_{j} t\right)\right\}(s) & =\frac{s}{s^{2}+\omega_{j}^{2}}, \\
\mathcal{L}\left\{\sin \left(\omega_{j} t\right)\right\}(s) & =\frac{\omega_{j}}{s^{2}+\omega_{j}^{2}} .
\end{aligned}
$$

With $s=-i \omega+0^{+}=-i\left(\omega+i 0^{+}\right)$we then easily obtain

$$
\begin{aligned}
\hat{q}_{\omega} & :=\mathcal{L}\{\hat{q}(t)\}\left(-i \omega+0^{+}\right) \\
& =\tilde{\chi}(\omega)\left[\hat{\xi}_{\omega}-i \omega M\{1+\tilde{\gamma}(\omega)\} \hat{q}(0)+M \dot{\hat{q}}(0)\right],
\end{aligned}
$$

where the Laplace-transformed damping kernel, the dynamic susceptibility, and the Laplace-transformed noise operator are, respectively, given by

$$
\begin{aligned}
& \tilde{\gamma}(\omega)=\frac{i \omega}{M} \sum_{j}^{N} \frac{c_{j}^{2}}{m_{j} \omega_{j}^{2}} \frac{1}{\omega^{2}-\omega_{j}^{2}}, \\
& \tilde{\chi}(\omega)=\frac{1}{M} \frac{1}{\omega_{0}^{2}-\omega^{2}-i \omega \tilde{\gamma}(\omega)}, \\
& \hat{\xi}_{\omega}=\sum_{j=1}^{N} \frac{c_{j}}{\omega^{2}-\omega_{j}^{2}}\left\{i \omega \hat{x}_{j}(0)-\frac{\hat{p}_{j}(0)}{m_{j}}\right\}-M \tilde{\gamma}(\omega) \hat{q}(0) .
\end{aligned}
$$

Substituting (11) into (12), we get

$$
\tilde{\chi}(\omega)=\frac{-\frac{1}{M} \prod_{j=1}^{N}\left(\omega^{2}-\omega_{j}^{2}\right)}{D_{\tilde{\chi}}(\omega)},
$$

where

$$
D_{\tilde{\chi}}(\omega)=\prod_{j=0}^{N}\left(\omega^{2}-\omega_{j}^{2}\right)-\frac{\omega^{2}}{M} \sum_{j=1}^{N} \frac{c_{j}^{2}}{m_{j} \omega_{j}^{2}} \prod_{\substack{j^{\prime}=1 \\(\neq j)}}^{N}\left(\omega^{2}-\omega_{j^{\prime}}^{2}\right) .
$$


It is known 15] that the susceptibility $\tilde{\chi}(\omega)$ in (12) has poles at the normal-mode frequencies of the total system $\hat{H}$ in (1), $\pm \bar{\omega}_{k}$ with $k=0,1,2, \cdots, N$, so that

$$
\omega_{0}^{2}-\bar{\omega}_{k}^{2}-i \bar{\omega}_{k} \tilde{\gamma}\left(\bar{\omega}_{k}\right)=0 .
$$

Here, we might be able to say that a specific $k=k_{0}$ would represent the "system harmonic oscillator" with the normal-mode frequency $\bar{\omega}_{k_{0}}$, uncoupled to the "bath" consisting of the remaining oscillators with $\bar{\omega}_{k}$, where $k \neq k_{0}$. From eqs. (12), (13), and (15), we have a compact expression of the susceptibility,

$$
\tilde{\chi}(\omega)=-\frac{1}{M} \frac{\prod_{j=1}^{N}\left(\omega^{2}-\omega_{j}^{2}\right)}{\prod_{k=0}^{N}\left(\omega^{2}-\bar{\omega}_{k}^{2}\right)} .
$$

Without any loss of generality, we here assume that

$$
\bar{\omega}_{0} \leq \bar{\omega}_{1} \leq \cdots \leq \bar{\omega}_{N-1} \leq \bar{\omega}_{N} .
$$

The damping function $\tilde{\gamma}(\omega)$ in the frequency domain has, besides eq. (11), another expression which is suitable for the case of a continuous distribution of bath modes; from eqs. (7) and (8) we obtain

$$
\begin{aligned}
& \tilde{\gamma}(\omega)=\frac{i}{M} \int_{0}^{\infty} \frac{d \omega^{\prime}}{\pi} \frac{J\left(\omega^{\prime}\right)}{\omega^{\prime}}\left(\frac{1}{\omega^{\prime}+\omega}-\frac{1}{\omega^{\prime}-\omega}\right), \\
& \left.\tilde{\gamma}(\omega)\right|_{\substack{\omega+i 0^{+} \\
\omega \rightarrow}}=\frac{J(\omega)}{M \omega}+ \\
& \quad \frac{i}{M} \int_{0}^{\infty} \frac{d \omega^{\prime}}{\pi} \frac{J\left(\omega^{\prime}\right)}{\omega^{\prime}} P\left(\frac{1}{\omega^{\prime}+\omega}-\frac{1}{\omega^{\prime}-\omega}\right) .
\end{aligned}
$$

We here used the well-known formula $1 /\left(x+i 0^{+}\right)=P(1 / x)-$ $i \pi \delta(x)$ for $x=\omega^{\prime}-\omega$. For the simple Ohmic case $J_{0}(\omega)=$ $M \gamma_{o} \omega$ with an $\omega$-independent constant $\gamma_{o}$, we easily have $\gamma_{0}(t)=2 \gamma_{o} \delta(t)$, and $\tilde{\gamma}_{0}(\omega)=\gamma_{o}$ with a vanishing principal (or imaginary) part in (19), while for the Drude model where $J_{d}(\omega)=M \gamma_{o} \omega \omega_{d}^{2} /\left(\omega^{2}+\omega_{d}^{2}\right)$ with a cut-off frequency $\omega_{d}$, we have $\gamma_{d}(t)=\gamma_{o} \omega_{d} e^{-\omega_{d} t}$, and

$$
\tilde{\gamma}_{d}(\omega)=\frac{\gamma_{o} \omega_{d}^{2}}{\omega^{2}+\omega_{d}^{2}}+i \frac{\gamma_{o} \omega_{d} \omega}{\omega^{2}+\omega_{d}^{2}}=\frac{\gamma_{o} \omega_{d}}{\omega_{d}-i \omega} .
$$

For a later purpose, it is interesting to compare $D_{\tilde{\chi}}(\omega)$ in eq. (14) with the denominator of the right hand side in (16). Then, we can easily find that

$$
\sum_{k=0}^{N} \bar{\omega}_{k}^{2}=\sum_{j=0}^{N} \omega_{j}^{2}+\gamma(0) ; \prod_{k=0}^{N} \bar{\omega}_{k}^{2}=\prod_{j=0}^{N} \omega_{j}^{2} .
$$

Here, $\gamma(0)=\left.\gamma(t)\right|_{t=0} \geq 0$ in eq. (50). From this comparison of the denominators at $\omega=\omega_{N}$, we also obtain $D_{\tilde{\chi}}\left(\omega_{N}\right) \leq 0$ and so $\omega_{N} \leq \bar{\omega}_{N}$. Similarly, we can acquire both $D_{\tilde{\chi}}\left(\omega_{1}\right) \leq 0$ for $N$ odd and $D_{\tilde{\chi}}\left(\omega_{1}\right) \geq 0$ for $N$ even, which lead to the fact that $\bar{\omega}_{0} \leq \omega_{1}$ for any given $N$. Further, we can obtain the relationship,
$D_{\tilde{\chi}}\left(\omega_{j}\right) \cdot D_{\tilde{\chi}}\left(\omega_{j-1}\right) \leq 0$ for any $j$. Therefore, it is found that

$$
\bar{\omega}_{0} \leq \omega_{1} \leq \bar{\omega}_{1} \leq \cdots \leq \omega_{N-1} \leq \bar{\omega}_{N-1} \leq \omega_{N} \leq \bar{\omega}_{N} .
$$

By using $D_{\tilde{\chi}}\left(\omega_{0}\right)$, we can also show that $\bar{\omega}_{0} \leq \omega_{0} \leq \bar{\omega}_{N}$ (see also [16]). Within this general treatment of the susceptibility, we would like to consider the quantum second law below.

\section{General validity of the quantum second law (discrete bath modes)}

The energy of the system oscillator $\hat{H}_{s}$ at zero temperature can be calculated by means of the partition function $Z=$ $\operatorname{Tr} e^{-\beta \hat{H}}$ with $\beta=1 / k_{B} T$ as

$$
\left\langle\hat{H}_{s}\right\rangle_{T=0}=\left.\frac{\operatorname{Tr}\left(\hat{H}_{s} e^{-\beta \hat{H}}\right)}{Z}\right|_{\beta \rightarrow \infty}=: E_{s}(0) .
$$

It is well-known [7,17, that the system-bath entanglement induced by the coupling term $\hat{H}_{s b}$ in (10) leads to the fact that the system oscillator $\hat{H}_{s}$, initially in a pure state (here, its ground state with the minimum energy $E_{g}=$ $\left.\hbar \omega_{0} / 2\right)$, is not in the pure state any longer but in a mixed state with a fluctuation in energy, and so we actually have $E_{s}(0)>E_{g}$. It was even discussed in [18] that the energy fluctuation measurements can provide entanglement information ( $c f$. for a thermodynamical approach to quantifying entanglement in bipartite qubit states, see [19]). From the fluctuation-dissipation theorem [12], we can also easily obtain

$$
E_{s}(0)=\frac{M \hbar}{2 \pi} \int_{0}^{\infty} d \omega\left(\omega_{0}^{2}+\omega^{2}\right) \operatorname{Im} \tilde{\chi}\left(\omega+i 0^{+}\right) .
$$

The factor $\operatorname{Im} \tilde{\chi}\left(\omega+i 0^{+}\right)$can be evaluated from eq. (16) with $\omega \rightarrow \omega+i 0^{+}$. By means of the technique used, e.g., in [20], eq. (24) can be rewritten as

$$
E_{s}(0)=\frac{\hbar}{2} \frac{1}{2 \pi i} \oint d \omega \frac{\omega_{0}^{2}+\omega^{2}}{G(\omega)},
$$

where $G(\omega)=-1 /\{M \tilde{\chi}(\omega)\}$, and the integration path is a loop around the positive real axis in the complex $\omega$ plane, consisting of the two branches, $(\infty+i \epsilon, i \epsilon)$ and $(-i \epsilon, \infty-i \epsilon)$. Therefore, $E_{s}(0)$ can be exactly obtained in closed form from the residues evaluated at all zeroes of $G(\omega)$ on the positive real axis. It is also interesting to note that the entanglement between any pair of the bath oscillators $\hat{H}_{j}=\hat{p}_{j}^{2} / 2 m_{j}+m_{j} \omega_{j}^{2} \hat{x}_{j}^{2} / 2$ with $j=1,2,3, \cdots, N$ is induced by the system-bath entanglement and the wellknown entanglement swapping. 21] As a result, we must obtain an excess energy for any $j$, i.e., $\left\langle\hat{H}_{j}\right\rangle_{T=0}>\hbar \omega_{j} / 2$. However, the energy of the total system, $\langle\hat{H}\rangle_{T=0}=\sum_{k=0}^{N} \hbar \bar{\omega}_{k} / 2$ is clearly not equivalent to $\left\langle\hat{H}_{s}\right\rangle_{T=0}+\sum_{j=1}^{N}\left\langle\hat{H}_{j}\right\rangle_{T=0}=$ $\left\langle\hat{H}_{s}+\hat{H}_{b}\right\rangle_{T=0}$. 
The minimum work required to couple a harmonic oscillator at temperature $T$ to a bath at the same temperature is equivalent to the Helmholtz free energy of the coupled total system minus the free energy of the uncoupled bath [1,11. The Helmholtz free energy can be obtained from the canonical partition function $Z_{s}(\beta)=$ $\operatorname{Tr} e^{-\beta \hat{H}} / \operatorname{Tr}_{b} e^{-\beta \hat{H}_{b}}$ as $F(T)=-k_{B} T \ln Z_{s}$, where $\operatorname{Tr}_{b}$ denotes the partial trace for the bath alone (in the absence of a coupling between system and bath, this would exactly correspond to the partition function of the system only). By means of the normal-mode frequencies $\bar{\omega}_{k}$ the partition function can be rewritten as

$$
Z_{s}(\beta)=\frac{\prod_{k=0} \sum_{n_{k}=0} e^{-\beta \hbar \bar{\omega}_{k}\left(n_{k}+\frac{1}{2}\right)}}{\prod_{j=1} \sum_{n_{j}=0} e^{-\beta \hbar \omega_{j}\left(n_{j}+\frac{1}{2}\right)}}
$$

so that we can easily get, for $\beta \rightarrow \infty$,

$$
F(0)=\frac{\hbar}{2}\left(\sum_{k=0}^{N} \bar{\omega}_{k}-\sum_{j=1}^{N} \omega_{j}\right) .
$$

With the aid of eq. (21), it is evidently found that $F(0)>$ $\hbar \omega_{j} / 2$ for any $j=0,1,2, \cdots, N$. Further, we have, from [22],

$$
F(T)=\frac{1}{\pi} \int_{0}^{\infty} d \omega f(\omega, T) \operatorname{Im}\left\{\frac{d}{d \omega} \ln \chi\left(\omega+i 0^{+}\right)\right\},
$$

where $f(\omega, T)=k_{B} T \ln \left\{2 \sinh \left(\hbar \omega / 2 k_{B} T\right)\right\}$. Similarly to eq. (25), we can obtain an integral form of the free energy at $T=0$,

$$
F(0)=\frac{\hbar}{2} \frac{1}{2 \pi i} \oint d \omega \frac{\omega G^{\prime}(\omega)}{G(\omega)} .
$$

Here, $f(\omega, 0)=\hbar \omega / 2$.

Now, we are in a position to exactly formulate the quantum second law within this general treatment; from eqs. (25) and (29) with (12), we easily find an expression

$$
K:=F(0)-E_{s}(0)=\frac{\hbar}{4 \pi} \oint d \omega \frac{\omega^{2} \tilde{\gamma}^{\prime}(\omega)}{G(\omega)},
$$

and, for the validity of the second law, we have to get $K \geq 0$ for any $N$ (the number of the bath oscillators) and the limit $N \rightarrow \infty$. Here, $K$ can exactly be evaluated from all residues of the integrand on the positive real axis. Substituting (16) with $\tilde{\chi}(\omega)=-1 / M G(\omega)$ and (11) into (30), we obtain, after a fairly lengthy evaluation of the contour integration (see Appendix $\mathrm{A}$ for details), the exact result

$$
K=\frac{\hbar}{8 M} \sum_{k=0}^{N} \mathcal{A}_{k}
$$

where

$$
\begin{aligned}
\mathcal{A}_{k}=\bar{\omega}_{k} \frac{\prod_{j=1}^{N}\left(\bar{\omega}_{k}^{2}-\omega_{j}^{2}\right)}{\prod_{\substack{k^{\prime}=0 \\
(\neq k)}}^{N}\left(\bar{\omega}_{k}^{2}-\bar{\omega}_{k^{\prime}}^{2}\right)} \times \\
\sum_{l=1}^{N} \frac{c_{l}^{2}}{m_{l} \omega_{l}^{2}} P\left\{\frac{1}{\left(\omega_{l}+\bar{\omega}_{k}\right)^{2}}+\frac{1}{\left(\omega_{l}-\bar{\omega}_{k}\right)^{2}}\right\} .
\end{aligned}
$$

Considering each summand $\mathcal{A}_{k}$ from $k=N$ with keeping in mind the frequency relationship in (22), we see that each of the summand is non-negative and so $K \geq 0$ indeed! Separately from this result for discrete bath modes, we will next discuss the second law for continuous bath modes. For doing this job, we will consider a continuation of the spectral density $J(\omega)$ from its original form in (6).

\section{The second law for continuous bath modes}

For a discussion of the second law for a continuous distribution of bath modes, we rewrite eq. (30) as

$$
K=\frac{\hbar}{4 \pi}\left(\int_{0}^{\infty} d \omega \frac{\omega^{2} \tilde{\gamma}_{-}^{\prime}(\omega)}{G_{-}(\omega)}-\int_{0}^{\infty} d \omega \frac{\omega^{2} \tilde{\gamma}_{+}^{\prime}(\omega)}{G_{+}(\omega)}\right)
$$

where the subscripts $+/-$ denote the branches $(\infty+i \epsilon, i \epsilon)$ and $(-i \epsilon, \infty-i \epsilon)$, respectively, so that

$$
\begin{aligned}
G_{+}(\omega):= & \left.G(\omega)\right|_{\substack{\omega \rightarrow+0^{+} \\
\omega+\omega^{+}}}=\omega^{2}-\omega_{0}^{2}+i \frac{J(\omega)}{M}- \\
& \frac{\omega}{M} \int_{0}^{\infty} \frac{d \omega^{\prime}}{\pi} \frac{J\left(\omega^{\prime}\right)}{\omega^{\prime}} P\left(\frac{1}{\omega^{\prime}+\omega}-\frac{1}{\omega^{\prime}-\omega}\right),
\end{aligned}
$$

and $G_{-}(\omega):=G(\omega) \mid \underset{\substack{\omega \rightarrow 0^{+} \\ \omega-i}}{\underset{\omega}{\omega}}=G_{+}^{*}(\omega)$. Here, we used $G(\omega)=$ $\omega^{2}-\omega_{0}^{2}+i \omega \tilde{\gamma}(\omega)$ with eq. (19) for $\tilde{\gamma}_{+}(\omega)$. Therefore, eq. (33) easily reduces to

$$
K=\frac{\hbar}{2 \pi} \operatorname{Im} \int_{0}^{\infty} d \omega \frac{\omega^{2} R_{+}^{\prime}(\omega)}{G_{+}(\omega)}
$$

where $R_{+}(\omega)=-i \tilde{\gamma}_{+}(\omega)$.

First, for the Ohmic case, $\left(\tilde{\gamma}_{+}\right)_{0}(\omega)=\gamma_{o}$, which is the prototype for damping, we easily obtain $K_{0}=0$. In fact, both $\left(E_{s}\right)_{0}(0)$ and $F_{0}(0)$ have the logarithmic divergence, however, the same value, namely,

$$
\left(E_{s}\right)_{0}(0)=F_{0}(0)=\frac{\hbar \gamma_{o}}{2 \pi} \int_{0}^{\infty} d \omega \frac{\omega\left(\omega^{2}+\omega_{0}^{2}\right)}{\left(\omega^{2}-\omega_{0}^{2}\right)^{2}+\left(\gamma_{o} \omega\right)^{2}}
$$

(see also the discussion in the last paragraphs of Secs. 4.1 and 4.2). However, the Ohmic model is not so realistic in its strict form because the spectral density of bath modes, $J_{0}(\omega)=M \gamma_{o} \omega$ diverges for large frequencies. We therefore introduce a cut-off frequency $\omega_{c}$ which leads to $J_{c}(\omega)$ decaying smoothly to zero for large frequencies $\omega>\omega_{c}$. We will first consider the Drude model, where $J_{d}(\omega)$ is 
polynomially decaying for $\omega>\omega_{c}=\omega_{d}$, and next a damping model with $J_{e}(\omega)$ being exponentially decaying for $\omega>\omega_{c}=\omega_{e}$. For these damping models, we will be able to show that $K>0$. Subsequently, we will also consider two different damping models without cut-off frequencies $\omega_{c}$; first, the extended Ohmic models where the spectral densities $J(\omega)$ diverge polynomially faster than $J_{0}(\omega)$, and secondly, the extended Drude models with $J_{d, n}(\omega)$ diverging faster or more slowly than $J_{0}(\omega)$. Interestingly, we will observe $K<0$ for some of the cut-off frequency-free damping models (see Secs. 4.3 and 4.4).

\subsection{Drude model $(d)$}

We briefly review the second law in the Drude model considered in [11; it is convenient to adopt, in place of $\left(\omega_{0}, \omega_{d}, \gamma_{o}\right)$, the parameters $\left(\mathbf{w}_{0}, \Omega, \gamma\right)$ through the relations

$$
\begin{gathered}
\omega_{0}^{2}:=\mathbf{w}_{0}^{2} \frac{\Omega}{\Omega+\gamma} ; \omega_{d}:=\Omega+\gamma \\
\gamma_{o}:=\gamma \frac{\Omega(\Omega+\gamma)+\mathbf{w}_{0}^{2}}{(\Omega+\gamma)^{2}} .
\end{gathered}
$$

Substituting eq. (20) with (37) into eq. (12), we obtain the susceptibility

$$
\begin{aligned}
& \tilde{\chi}_{d}(\omega) \\
= & -\frac{1}{M} \frac{\omega+i \omega_{d}}{\omega^{3}+i \omega_{d} \omega^{2}-\left(\omega_{0}^{2}+\gamma_{o} \omega_{d}\right) \omega-i \omega_{0}^{2} \omega_{d}} \\
= & -\frac{1}{M} \frac{\omega+i\left(\Omega+z_{1}+z_{2}\right)}{(\omega+i \Omega)\left(\omega+i z_{1}\right)\left(\omega+i z_{2}\right)}
\end{aligned}
$$

where $z_{1}=\gamma / 2+i \mathbf{w}_{1}$ and $z_{2}=\gamma / 2-i \mathbf{w}_{1}$ with $\mathbf{w}_{1}^{2}=$ $\mathbf{w}_{0}^{2}-(\gamma / 2)^{2}$. This gives us $\left(G_{+}\right)_{d}(\omega)=-1 /\left\{M \tilde{\chi}_{d}(\omega)\right\}$ for eq. (35). By means of eq. (39), we can even obtain the closed expressions for both $\left(E_{s}\right)_{d}(0)$ from (24) and $F_{d}(0)$ from (28). We give the detailed derivation of these expressions in Appendix B, which will also be used in Sec. 4.4 It has been numerically shown in [11 that $\left(E_{s}\right)_{d}(0)$ in (65) is actually greater than $E_{g}=\frac{\hbar \mathbf{w}_{0}}{2} \sqrt{\frac{\Omega}{\Omega+\gamma}}$, and $F_{d}(0)$ in (68) is even greater than the $\left(E_{s}\right)_{d}(0)$, i.e., $K_{d}>0$. For a later purpose, we will also evaluate $K_{d}$ explicitly for various pairs $\left(\omega_{0}, \omega_{d}\right)$ (see Table 2 in Sec. 4.4).

It is noted that in the limit $\omega_{d} \rightarrow \infty$ (equivalently, $\Omega \rightarrow \infty$ ), we have $K_{d} \rightarrow \frac{\gamma}{\pi \mathbf{w}_{0}} E_{g}$ (see Appendix B). From the comparison between $\tilde{\gamma}_{d}(\omega)$ and $\tilde{\gamma}_{0}(\omega)$ (or, equivalently, $J_{d}(\omega)$ and $J_{0}(\omega)$ ), this result would be interpreted as $K_{0} \rightarrow$ $\frac{\gamma}{\pi \mathbf{w}_{0}} E_{g}$. However, it is misleading; $\tilde{\gamma}_{d}(\omega)$ behaves only for small frequencies, $\omega \ll \omega_{d}$, like in the Ohmic case, which corresponds to $\gamma_{d}(t) \rightarrow \gamma_{0}(t)$ only for large times. Actually, $\gamma_{d}(t)=\gamma_{o} \omega_{d} e^{-\omega_{d} t}$ with $\omega_{d} \rightarrow \infty$ does not reduce to $\gamma_{0}(t)=2 \gamma_{o} \delta(t)=\lim _{\omega_{d} \rightarrow \infty} \frac{2}{\sqrt{\pi}} \gamma_{o} \sqrt{\omega_{d}} e^{-\omega_{d} t^{2}}$. For the evaluation of $K$, however, all frequencies, $0 \leq$ $\omega<\infty$, have to be considered. Therefore, we evidently get $\lim _{\omega_{d} \rightarrow \infty} K_{d} \gtrless K_{0}=0$.

\subsection{Exponentially decaying model $(e)$}

We now consider a damping model with $J_{e}(\omega)=M \gamma_{o} \omega e^{-\omega / \omega_{e}}$ which, in the limit $\omega_{e} \rightarrow \infty$, clearly reduces to $J_{0}(\omega)$ for small frequencies. Substituting this into eq. (7), we can obtain

$$
\gamma_{e}(t)=\frac{2}{\pi} \frac{\gamma_{o} \omega_{e}}{1+\left(\omega_{e} t\right)^{2}}
$$

Applying the Laplace transform [14] to eq. (40) with $s=$ $-i \omega+0^{+}$, it can be found that

$$
\begin{aligned}
\tilde{\gamma}_{e}(\omega)= & \gamma_{o} e^{-\omega / \omega_{e}}+ \\
& i \frac{\gamma_{o}}{\pi}\left\{e^{\omega / \omega_{e}} E_{1}\left(\frac{\omega}{\omega_{e}}\right)+e^{-\omega / \omega_{e}} \operatorname{Ei}\left(\frac{\omega}{\omega_{e}}\right)\right\},
\end{aligned}
$$

(see Appendix $\mathrm{C}$ for the detailed derivation). By using this with $E_{1}^{\prime}(y)=-E_{0}(y)=-e^{-y} / y$, we can easily get $\left(R_{+}^{\prime}\right)_{e}(\omega)$ and $\left(G_{+}\right)_{e}(\omega)$, and then introducing a dimensionless variable $\lambda=\omega / \omega_{e}$, we arrive at the expression

$$
K_{e}=\frac{\hbar \gamma_{o} \omega_{e}^{2}}{2 \pi^{2}} \operatorname{Im} \int_{0}^{\infty} d \lambda \frac{f_{1}(\lambda)}{f_{2}(\lambda)}
$$

where

$$
\begin{aligned}
f_{1}(\lambda)= & \lambda^{2}\left\{e^{\lambda} E_{1}(\lambda)-e^{-\lambda} \operatorname{Ei}(\lambda)+i \pi e^{-\lambda}\right\}, \\
f_{2}(\lambda)= & \omega_{e}^{2} \lambda^{2}-\omega_{0}^{2}-\frac{\gamma_{o} \omega_{e}}{\pi} \lambda\left\{e^{\lambda} E_{1}(\lambda)+e^{-\lambda} \operatorname{Ei}(\lambda)\right\} \\
& +i \omega_{e} \gamma_{o} \lambda e^{-\lambda} .
\end{aligned}
$$

We numerically evaluate the integration in (42) for various pairs $\left(\gamma_{o}, \omega_{e}\right)$ to show that $K_{e}>0$ (see Table 1).

From the fact that $\tilde{\gamma}_{e}(\omega)$ behaves like in the Ohmic case for small frequencies $\omega \ll \omega_{e}$, it is also interesting to consider the leading behavior of $K_{e}$ for $\omega_{e} \rightarrow \infty$; from eq. (42) we can easily get $\lim _{\omega_{e} \rightarrow \infty} K_{e}=\frac{\hbar \gamma_{o}}{2 \pi} \neq 0$, which is also different from $\lim _{\omega_{d} \rightarrow \infty} K_{d}$ in Sec. 4.1 This confirms that these limiting values cannot reveal the Ohmic counterpart $K_{0}$.

Table 1. $K_{e} / E_{g}$ for various pairs $\left(\gamma_{o}, \omega_{e}\right)$, where $E_{g}=\frac{\hbar}{2}$ (i.e., $\left.\omega_{0}=1\right) ; \lim _{\omega_{e} \rightarrow \infty} K_{e} / E_{g}=\gamma_{o} / \pi$.

\begin{tabular}{|c||cccc|}
\hline$\omega_{e}$ & $\gamma_{o}=0.5$ & $\gamma_{o}=1$ & $\gamma_{o}=2$ & $\gamma_{o}=5$ \\
\hline 0.5 & 0.04225 & 0.08186 & 0.15604 & 0.34038 \\
1 & 0.06130 & 0.11838 & 0.22117 & 0.47348 \\
5 & 0.10600 & 0.20348 & 0.37899 & 0.81614 \\
10 & 0.12131 & 0.23326 & 0.43819 & 0.96224 \\
50 & 0.14414 & 0.28018 & 0.54302 & 1.25567 \\
80 & 0.14789 & 0.28896 & 0.56377 & 1.32020 \\
\hline \hline$\infty$ & 0.15915 & 0.31831 & 0.63662 & 1.59155 \\
\hline
\end{tabular}

\subsection{Extended Ohmic models $(p)$}

Let us consider damping models with $J_{p}(\omega)=M \gamma_{o} \omega\left(\omega / \gamma_{o}\right)^{p}$ being polynomially divergent with $\omega$. Clearly, the case of 
$p=0$ is Ohmic. First, we have $J_{1}(\omega)=M \omega^{2}$. By using the relationship $\int_{0}^{\infty} d y e^{i k y}=\pi \delta(k)+i P(1 / k)$, we can easily obtain $\gamma_{1}(t)=-\frac{2}{\pi} P \frac{1}{t^{2}}$, which leads to no welldefined $\tilde{\gamma}_{1}(\omega)$. This $(p=1)$ is, therefore, physically not acceptable. It is not difficult to show that the cases of $p$ being odd are not acceptable.

Next, we consider the case of $p=2$. It can be shown that $\gamma_{2}(t)=-\frac{2}{\gamma_{o}} \delta^{\prime \prime}(t)$ and $\left(\tilde{\gamma}_{+}\right)_{2}(\omega)=\frac{\omega^{2}}{\gamma_{o}}-i \frac{2 \delta(0)}{\gamma_{o}} \omega$. By using this for eq. (35), we can obtain

$$
K_{2}=\frac{\hbar}{2 \pi} \operatorname{Im} \int_{0}^{\infty} d \omega \frac{2 \omega^{2}\{-\omega+i \delta(0)\}}{\omega^{3}-i \alpha \omega^{2}+i \beta},
$$

where $\alpha=\gamma_{o}+2 \delta(0)$ and $\beta=\omega_{0}^{2} \gamma_{o}$. The integral in (45) diverges logarithmically. This divergence is, obviously, from the fact that both $\left(E_{s}\right)_{2}(0)$ and $F_{2}(0)$ diverge logarithmically, however, differently from the Ohmic case, $\left(E_{s}\right)_{2}(0) \neq$ $F_{2}(0)$. In fact, we find that $K_{2}=-\frac{\hbar}{\pi}\left\{\delta(0)+\gamma_{o}\right\} \times \infty<0$, which clearly means that the excess energy, $\left(E_{s}\right)_{2}(0)$, is greater than the minimum work (or the work in a reversible process), $F_{2}(0)$, required to couple a system to a bath. This violation of the second law in the reversible process may be understood to emerge from a large amount of the energy offer by the bath with $J_{2}(\omega)$ diverging with $\omega$. The infinite value of $K_{2}$ suggests, however, that this model would be strictly unrealistic.

\subsection{Extended Drude models $(d, n)$}

We now consider a more general class of the spectral density than $J_{d}(\omega)$, which is

$$
J_{d, n}(\omega)=\left(\frac{\omega}{\omega_{d}}\right)^{n} J_{d}(\omega)=M \gamma_{o} \frac{\omega^{n+1}}{\omega_{d}^{n-2}\left(\omega^{2}+\omega_{d}^{2}\right)} .
$$

Let us begin with $n$ being odd. First, $n=1$. We then have $J_{d, 1}(\omega)=M \gamma_{o} \omega_{d} \omega^{2} /\left(\omega^{2}+\omega_{d}^{2}\right)$, which converges to a nonzero constant $M \gamma_{o} \omega_{d}$ for large frequencies. Substituting this into eq. (7), we can obtain, after some calculation (see Appendix C for details),

$$
\begin{aligned}
\gamma_{d, 1}(t)= & \frac{\gamma_{o} \omega_{d}}{\pi}\left[\left\{\operatorname{Ei}\left(\omega_{d} t\right)+E_{1}\left(\omega_{d} t\right)\right\} \sinh \left(\omega_{d} t\right)-\right. \\
& \left.\left\{\operatorname{Ei}\left(\omega_{d} t\right)-E_{1}\left(\omega_{d} t\right)\right\} \cosh \left(\omega_{d} t\right)\right] .
\end{aligned}
$$

Applying the Laplace transform [14] to this, we can get

$$
\left(\tilde{\gamma}_{+}\right)_{d, 1}(\omega)=\frac{\gamma_{o} \omega_{d} \omega}{\omega^{2}+\omega_{d}^{2}}+i \frac{2}{\pi} \frac{\gamma_{o} \omega_{d} \omega}{\omega^{2}+\omega_{d}^{2}} \ln \left(\frac{\omega}{\omega_{d}}\right) .
$$

Using eq. (34) with (48), we can arrive at the expression in (35)

$$
K_{d, 1}=\frac{\hbar \gamma_{o}}{2 \pi} \operatorname{Im} \int_{0}^{\infty} d \lambda \frac{g_{1}(\lambda)}{g_{2}(\lambda)}
$$

where

$$
\begin{aligned}
g_{1}(\lambda)= & \lambda^{2}\left[\frac{2 \lambda_{d}}{\pi}\left\{\left(\lambda_{d}^{2}-\lambda^{2}\right) \ln \left(\frac{\lambda}{\lambda_{d}}\right)+\lambda^{2}+\lambda_{d}^{2}\right\}+\right. \\
& \left.i \lambda_{d}\left(\lambda^{2}-\lambda_{d}^{2}\right)\right] \\
g_{2}(\lambda)= & \left(\lambda^{2}+\lambda_{d}^{2}\right)\left\{\left(\lambda^{2}-\lambda_{0}^{2}\right)\left(\lambda^{2}+\lambda_{d}^{2}\right)-\right. \\
& \left.\frac{2 \lambda_{d} \lambda^{2}}{\pi} \ln \left(\frac{\lambda}{\lambda_{d}}\right)+i \lambda_{d} \lambda^{2}\right\}
\end{aligned}
$$

Here, we introduced a dimensionless variable $\lambda=\omega / \gamma_{o}$ with $\lambda_{0}=\omega_{0} / \gamma_{o}$ and $\lambda_{d}=\omega_{d} / \gamma_{o}$. We numerically evaluate $K_{d, 1}$ for various pairs $\left(\lambda_{0}, \lambda_{d}\right)$ to show that $K_{d, 1}>0$ (see Table 2). It is also noted that in the limit $\lambda_{d}$ (or $\left.\omega_{d}\right)$ $\rightarrow \infty$, the spectral density $J_{d, 1}(\omega)$ with $\gamma_{o}=\omega_{d}$ reduces to $J_{1}(\omega)$ in Sec. 4.3 for small frequencies. As was discussed, however, this case $(d, 1)$ is a well-defined damping model whereas the model with $J_{1}(\omega)$ is not.

Table 2. $K_{d} \pi / \gamma_{o} E_{g}$ from (71) versus $K_{d, 1} \pi / \gamma_{o} E_{g}$ from (49) for various pairs $\left(\omega_{0}, \omega_{d}\right)$ with $\gamma_{o}=1$, where $E_{g}=\frac{\hbar \omega_{0}}{2} ;(d, 0)$ denotes the Drude model.

\begin{tabular}{|c|c|c||c|c|c|}
\hline$\left(\omega_{0}, \omega_{d}\right)$ & $(d, 0)$ & $(d, 1)$ & $\left(\omega_{0}, \omega_{d}\right)$ & $(d, 0)$ & $(d, 1)$ \\
\hline \hline$(0.5,0.5)$ & 0.84275 & 0.50184 & $(1,0.5)$ & 1.21124 & 0.25468 \\
\hline$(0.5,1)$ & 0.61942 & 0.45203 & $(1,1)$ & 0.45318 & 0.26521 \\
\hline$(0.5,5)$ & 1.29483 & 0.34454 & $(1,5)$ & 0.61427 & 0.17800 \\
\hline$(0.5,10)$ & 1.52266 & 0.24507 & $(1,10)$ & 0.73767 & 0.05454 \\
\hline \hline$(5,0.5)$ & 0.53089 & 0.02832 & $(10,0.5)$ & 0.28968 & 0.00936 \\
\hline$(5,1)$ & 0.47010 & 0.04106 & $(10,1)$ & 0.27637 & 0.01345 \\
\hline$(5,5)$ & 0.09790 & 0.05476 & $(10,5)$ & 0.13428 & 0.02346 \\
\hline$(5,10)$ & 0.09537 & 0.04976 & $(10,10)$ & 0.04792 & 0.02347 \\
\hline
\end{tabular}

Let $n=3$ next. We have $J(\omega)_{d, 3}=M \gamma_{o} \omega_{d}^{-1} \omega^{4} /\left(\omega^{2}+\right.$ $\left.\omega_{d}^{2}\right)$. After a straightforward calculation, we will obtain

$$
\gamma_{d, 3}(t)=-\gamma_{d, 1}(t)-\frac{2 \gamma_{o}}{\pi \omega_{d}} \frac{P}{t^{2}}
$$

which indicates that this case is physically not acceptable. Similarly, we can also show that all cases for $n>3$ being odd are not acceptable.

Now, let $n$ be even. We can then find that

$$
\gamma_{d, 2 m}(t)=(-1)^{m}\left\{\gamma_{d}(t)-\sum_{j=1}^{m} \frac{\gamma_{0}^{\{2(j-1)\}}(t)}{\omega_{d}^{2(j-1)}}\right\}
$$

where $m=1,2, \cdots$, and $\gamma_{0}^{\{2(j-1)\}}(t)$ represent $2(j-1)$ time derivatives of $\gamma_{0}(t)$. We begin with a simple case $(m=1)$ with $J_{d, 2}(\omega)=M \gamma_{o} \omega^{3} /\left(\omega^{2}+\omega_{d}^{2}\right)$. This case is particularly interesting because $J_{d, 2}(\omega)$ diverges for large frequencies, however, more slowly than $J_{0}(\omega)$ for the Ohmic case, whereas all $J_{d, 2 m}(\omega)$ for $m>1$ diverge faster than $J_{0}(\omega) ; J_{d, 2}(\omega)$ may be said to be of weak divergence. Due to the fact that $K_{d, 0}>K_{d, 1}>0$ seen from Table 2 , we would like to pose a question if we will here obtain $K_{d, 1}>K_{d, 2}>0=K_{0}$ or $K_{d, 1}>0 \geq K_{d, 2}$. In fact, we have an interesting relation $\left(\tilde{\gamma}_{+}\right)_{d, 2}(\omega)=-\tilde{\gamma}_{d}(\omega)+\tilde{\gamma}_{0}(\omega)$ from eq. (52), and so $\left(R_{+}^{\prime}\right)_{d, 2}(\omega)=-\left(R_{+}^{\prime}\right)_{d}(\omega)$ for eq. (35). Introducing the parameters $\left(\mathbf{w}_{0}, \Omega, \gamma\right)$ defined as the relations

$\omega_{d} \omega_{0}^{2}:=\Omega \mathbf{w}_{0}^{2} ; \omega_{d}+\gamma_{o}:=\Omega+\gamma ; \omega_{0}^{2}:=\Omega \gamma+\mathbf{w}_{0}^{2}$ 
(note that these differ from the relations in (37)), we can easily obtain

$$
\begin{aligned}
\left(\tilde{G}_{+}\right)_{d, 2}(\omega) & =\frac{\omega^{3}+i\left(\gamma_{o}+\omega_{d}\right) \omega^{2}-\omega_{0}^{2} \omega-i \omega_{0}^{2} \omega_{d}}{\omega+i \omega_{d}} \\
& =\frac{(\omega+i \Omega)\left(\omega+i z_{1}\right)\left(\omega+i z_{2}\right)}{\omega+i \Omega \mathbf{w}_{0}^{2} /\left(\Omega \gamma+\mathbf{w}_{0}^{2}\right)}
\end{aligned}
$$

where $z_{1}=\frac{\gamma}{2}+i \mathbf{w}_{1}$ and $z_{2}=\frac{\gamma}{2}-i \mathbf{w}_{1}$ with $\mathbf{w}_{1}^{2}=$ $\mathbf{w}_{0}^{2}-\left(\frac{\gamma}{2}\right)^{2}$. By using eqs. (35) and (54), we arrive at the expression

$$
K_{d, 2}=\frac{\hbar}{2 \pi} \frac{\Omega \mathbf{w}_{0}^{2}}{\left(\Omega \gamma+\mathbf{w}_{0}^{2}\right)\left(\Omega \gamma-\Omega^{2}-\mathbf{w}_{0}^{2}\right)} C\left(\mathbf{w}_{0}, \Omega, \gamma\right)
$$

where

$$
\begin{aligned}
C\left(\mathbf{w}_{0}, \Omega, \gamma\right)= & \gamma\left(\mathbf{w}_{0}^{2}-\Omega^{2}\right) \frac{1}{\mathbf{w}_{1}} \arctan \frac{2 \mathbf{w}_{1}}{\gamma}+ \\
& \left(\Omega^{2}+\mathbf{w}_{0}^{2}-\Omega \gamma\right) \ln \left(\Omega \gamma+\mathbf{w}_{0}^{2}\right)- \\
& 2\left(\Omega^{2}+\mathbf{w}_{0}^{2}\right) \ln \mathbf{w}_{0}+2 \Omega \gamma \ln \Omega
\end{aligned}
$$

In case that $\mathbf{w}_{1}$ is complex-valued $\left(\mathbf{w}_{0}<\gamma / 2\right)$, i.e., for the overdamped case, this has to be understood in terms of the relation, $\frac{1}{\mathbf{w}_{1}} \arctan \frac{2 \mathbf{w}_{1}}{\gamma}=\frac{1}{2 \overline{\mathbf{w}}_{1}} \ln \left(\frac{\gamma+2 \overline{\mathbf{w}}_{1}}{\gamma-2 \overline{\mathbf{w}}_{1}}\right)$, where $\overline{\mathbf{w}}_{1}^{2}=\left(\frac{\gamma}{2}\right)^{2}-\mathbf{w}_{0}^{2}$.

Interestingly enough, we can here observe $K_{d, 2}<0$ (see Fig. 1), which would allow us to have a violation of the second law in the reversible process for this damping model. This negativity may be understood from the comparison, with the aid of the relation $\left(R_{+}^{\prime}\right)_{d, 2}(\omega)=$ $-\left(R_{+}^{\prime}\right)_{d}(\omega)$, between

$$
\begin{aligned}
K_{d, 2}= & \frac{\hbar}{2 \pi} \omega_{d} \gamma_{o} \times \\
& \operatorname{Im} \int_{0}^{\infty} d \omega \frac{\omega^{2}}{\left(\omega+i \omega_{d}\right)(\omega+i \Omega)\left(\omega+i z_{1}\right)\left(\omega+i z_{2}\right)}
\end{aligned}
$$

with $\left(\mathbf{w}_{0}, \Omega, \gamma\right)$ in eq. (37) and $\omega_{d}=\Omega+\gamma$, and

$$
\begin{aligned}
K_{d}= & -\frac{\hbar}{2 \pi} \omega_{d} \gamma_{o} \times \\
& \operatorname{Im} \int_{0}^{\infty} d \omega \frac{\omega^{2}}{\left(\omega+i \omega_{d}\right)(\omega+i \Omega)\left(\omega+i z_{1}\right)\left(\omega+i z_{2}\right)}>0
\end{aligned}
$$

with $\left(\mathbf{w}_{0}, \Omega, \gamma\right)$ in (53) and $\omega_{d}=\mathbf{w}_{0}^{2} \Omega /\left(\Omega \gamma+\mathbf{w}_{0}^{2}\right)$. In fact, we can also show, by using eq. (28) with (54), that $F_{d, 2}(0)>F_{d}(0)$.

Next, we briefly consider the case of $(d, 4)$. We then have $\left(\tilde{\gamma}_{+}\right)_{d, 4}(\omega)=\tilde{\gamma}_{d}(\omega)-\gamma_{o}+\left(\gamma_{o} \omega^{2}-2 i \gamma_{o} \delta(0) \omega\right) / \omega_{d}^{2}$ from eq. (52). After a fairly lengthy calculation with this, we can eventually obtain an explicit expression for $K_{d, 4}=$ $\frac{\hbar}{2 \pi} \int_{0}^{\infty} d \omega f_{d, 4}(\omega)$, where $f_{d, 4}(\omega) \rightarrow-2\left\{\delta(0)+\omega_{d}^{2} / \gamma_{o}\right\} / \omega$ for large $\omega$. From this asymptotic form, we easily see that $K_{d, 4} \rightarrow-\infty$, which indicates the violation of the second law. This infinity of $K_{d, 4}$ suggests, however, that this case would be strictly unrealistic.

From the above results for cases $(d, n)$ including the case $(p=2)$ in Sec. 4.3, we may be able to say that aside for physically unacceptable damping models, the divergence (weak or strict) of the spectral density $J(\omega)$ for large frequencies could lead to the violation of the second law.
It is also interesting here to note that $\operatorname{Re}\left(\tilde{\gamma}_{+}\right)_{d, 2}(\omega)=$ $\gamma_{o} \omega^{2} /\left(\omega^{2}+\omega_{d}^{2}\right)>0$ and $\operatorname{Re}\left(\tilde{\gamma}_{+}\right)_{d, 4}(\omega)=\gamma_{o} \omega_{d}^{-2} \omega^{4} /\left(\omega^{2}+\right.$ $\left.\omega_{d}^{2}\right)>0$. It is known [23] that a violation of the positivity for $\operatorname{Re} \tilde{\gamma}_{+}(\omega)$ is tantamount to a violation of the second law in the thermodynamic limit (where a coupling strength between system and bath vanishes). We see here, however, that this positivity would not be a sufficient condition for the second law in the quantum regime (with a non-negligible finite coupling strength between system and bath).

\section{Conclusions}

In summary, we have extensively studied the second law in the scheme of quantum Brownian motion. It has been observed that from the system-bath entanglement, a system oscillator coupled to a bath at zero temperature has a higher average energy value than the ground state of an uncoupled harmonic oscillator. For a damping model with arbitrarily discrete bath modes and damping models with continuous bath modes with cut-off frequencies, however, this apparent excess energy has actually been found to be less than the minimum work to couple a system to a bath. Therefore, the second law holds in the quantum regime. We also found, on the other hand, that the violation of the second law may happen for some cutoff frequency-free damping models, which are, however, physically unrealistic; especially the case $(d, 2)$ in Sec.4.4, with a less diverging spectral density of bath modes than the Ohmic model being the prototype for damping, has a finitely negative value of $K_{d, 2}$. The further question about the validity of the quantum second law for a broader class of quantum systems than the quantum Brownian motion considered here, particularly non-linear systems coupled to a bath, clearly remains open.

\section{Acknowledgments}

One of us (I. K.) is grateful to G.J. Iafrate for some interesting remarks. 


\section{A : A detailed derivation of eq. (31)}

By substituting eq. (16) with $\tilde{\chi}(\omega)=-1 / M G(\omega)$ and eq. (11) into $K$ in (30), we immediately have

$$
K=\frac{-i \hbar}{4 \pi M} \oint d \omega \omega^{2} \frac{\prod_{j=1}^{N}\left(\omega^{2}-\omega_{j}^{2}\right)}{\prod_{k=0}^{N}\left(\omega^{2}-\bar{\omega}_{k}^{2}\right)} \sum_{l=1}^{N} \frac{c_{l}^{2}}{2 m_{l} \omega_{l}^{2}}\left\{\frac{1}{\left(\omega_{l}+\omega\right)^{2}}+\frac{1}{\left(\omega_{l}-\omega\right)^{2}}\right\}
$$

where the integration path over $\omega$ is a loop around the positive real axis in the complex $\omega$-plane, consisting of the two branches, $(\infty+i \epsilon, i \epsilon)$ and $(-i \epsilon, \infty-i \epsilon)$. By using the residues at all poles $\omega=\bar{\omega}_{k}$ of the integrand, we can evaluate the contour integration. In doing so, we do not have any residue at $\omega=\bar{\omega}_{k^{\prime}}$ when $\omega_{l}=\bar{\omega}_{k^{\prime}}$. Accordingly, we can finally obtain

$$
K=\frac{\hbar}{4 M} \sum_{k=0}^{N} \mathcal{A}_{k} ; \mathcal{A}_{k}=\mathcal{A}_{k}^{(1)} \cdot \mathcal{A}_{k}^{(2)},
$$

where

$$
\mathcal{A}_{k}^{(1)}=\bar{\omega}_{k} \frac{\prod_{j=1}^{N}\left(\bar{\omega}_{k}^{2}-\omega_{j}^{2}\right)}{\prod_{\substack{k^{\prime}=0 \\(\neq k)}}^{N}\left(\bar{\omega}_{k}^{2}-\bar{\omega}_{k^{\prime}}^{2}\right)} ; \mathcal{A}_{k}^{(2)}=\sum_{l=1}^{N} \frac{c_{l}^{2}}{2 m_{l} \omega_{l}^{2}} P\left\{\frac{1}{\left(\omega_{l}+\bar{\omega}_{k}\right)^{2}}+\frac{1}{\left(\omega_{l}-\bar{\omega}_{k}\right)^{2}}\right\} .
$$

Here, $\mathcal{A}_{k}^{(2)}$ is, obviously, positive-valued. Therefore, the non-negativeness of $\mathcal{A}_{k}$ can be completely determined by the factor $\mathcal{A}_{k}^{(1)}$. Keeping in mind the frequency relationship in (22), we first consider $\mathcal{A}_{N}^{(1)}$. This is clearly non-negative. Next, for $k=N-1$, we have

$$
\mathcal{A}_{N-1}^{(1)}=\bar{\omega}_{N-1} \frac{\prod_{j=1}^{N-1}\left(\bar{\omega}_{N-1}^{2}-\omega_{j}^{2}\right)}{\prod_{k=0}^{N-2}\left(\bar{\omega}_{N-1}^{2}-\bar{\omega}_{k}^{2}\right)} \times \frac{\bar{\omega}_{N-1}^{2}-\omega_{N}^{2}}{\bar{\omega}_{N-1}^{2}-\bar{\omega}_{N}^{2}}
$$

The first factor on the right hand side is non-negative, and so is the second factor whose numerator and denominator are negative-valued, respectively. Therefore, we get $\mathcal{A}_{N-1} \geq 0$. Along the same line, we can straightforwardly show that each summand $\mathcal{A}_{k}$ with $k=N-2, N-3, \cdots, 0$ is non-negative, which will yield $K \geq 0$.

\section{B : No violation of the second law in the Drude model}

In the Drude model, we can even evaluate the system energy $\left(E_{s}\right)_{d}(0)$ and the free energy $F_{d}(0)$ explicitly and in closed form. From eq. (39) we see that for $\mathbf{w}_{0}>\gamma / 2$ (underdamped case), $z_{1}$ and $z_{2}$ are conjugate complex numbers to each other, while for $\mathbf{w}_{0} \leq \gamma / 2$ (overdamped case), both $z_{1}$ and $z_{2}$ are real-valued. Therefore, $\operatorname{Im}\left\{\left(R_{+}^{\prime}\right)_{d}(\omega) /\left(G_{+}\right)_{d}(\omega)\right\}$ in (35), and thus the explicit expressions of $K_{d}$, for both cases would differ from each other in parameters $\left(\mathbf{w}_{0}, \Omega, \gamma\right)$. By using eq. (39) we can obtain

$$
\begin{aligned}
& \int_{0}^{\infty} d \omega \operatorname{Im} \chi_{d}(\omega)= \begin{cases}\frac{1}{M} \frac{\left(\mathbf{w}_{0}^{2}+\Omega^{2}-\gamma^{2} / 2\right) \arccos \left(\gamma / 2 \mathbf{w}_{0}\right)-\gamma \mathbf{w}_{1} \ln \left(\Omega / \mathbf{w}_{0}\right)}{\mathbf{w}_{1}\left(\mathbf{w}_{0}^{2}-\Omega \gamma+\Omega^{2}\right)} & \text { for } \quad \mathbf{w}_{0}>\gamma / 2 \\
\frac{1}{M} \frac{\left(\gamma^{2} / 2-\mathbf{w}_{0}^{2}-\Omega^{2}\right) / 2 \cdot \ln \left(\frac{\gamma / 2-\overline{\mathbf{w}}_{1}}{\gamma / 2+\overline{\mathbf{w}}_{1}}\right)-\gamma \overline{\mathbf{w}}_{1} \ln \left(\Omega / \mathbf{w}_{0}\right)}{\overline{\mathbf{w}}_{1}\left(\mathbf{w}_{0}^{2}-\Omega \gamma+\Omega^{2}\right)} & \text { for } \quad \mathbf{w}_{0} \leq \gamma / 2\end{cases}
\end{aligned}
$$

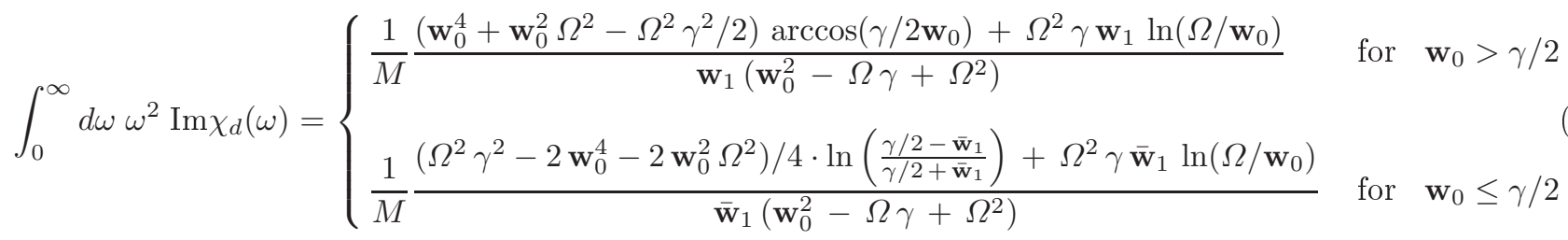


where $\mathbf{w}_{1}=\sqrt{\mathbf{w}_{0}^{2}-(\gamma / 2)^{2}}$ and $\overline{\mathbf{w}}_{1}=-i \mathbf{w}_{1}$.

From eqs. (24), (63), and (64), we have an exact expression

$$
\left(E_{s}\right)_{d}(0)=\frac{\hbar}{2 \pi}\left\{A\left(\mathbf{w}_{0}, \Omega, \gamma\right)+B\left(\mathbf{w}_{0}, \Omega, \gamma\right)\right\},
$$

where

$$
A\left(\mathbf{w}_{0}, \Omega, \gamma\right)=\left\{\begin{array}{l}
\frac{\left(\mathbf{w}_{0}^{2}+\Omega^{2}\right)\left(2 \Omega \mathbf{w}_{1}^{2}+\mathbf{w}_{0}^{2} \gamma\right)-\Omega^{2} \gamma^{3} / 2}{\mathbf{w}_{1}(\Omega+\gamma)\left(\mathbf{w}_{0}^{2}-\Omega \gamma+\Omega^{2}\right)} \arccos \left(\gamma / 2 \mathbf{w}_{0}\right) \\
\frac{\left(\mathbf{w}_{0}^{2}+\Omega^{2}\right)\left(\Omega \gamma^{2} / 4-\mathbf{w}_{0}^{3}-\mathbf{w}_{0}^{2} \gamma / 2\right)-\Omega \gamma^{2} / 2 \cdot\left(\mathbf{w}_{0}^{2}-\Omega \gamma / 2\right)}{\overline{\mathbf{w}}_{1}(\Omega+\gamma)\left(\mathbf{w}_{0}^{2}-\Omega \gamma+\Omega^{2}\right)} \ln \left(\frac{\gamma / 2-\overline{\mathbf{w}}_{1}}{\gamma / 2+\overline{\mathbf{w}}_{1}}\right)
\end{array}\right.
$$

for $\mathbf{w}_{0}>\gamma / 2$ and $\mathbf{w}_{0} \leq \gamma / 2$, respectively, and

$$
B\left(\mathbf{w}_{0}, \Omega, \gamma\right)=\frac{\Omega \gamma\left(\Omega^{2}+\Omega \gamma-\mathbf{w}_{0}^{2}\right)}{(\Omega+\gamma)\left(\mathbf{w}_{0}^{2}-\Omega \gamma+\Omega^{2}\right)} \ln \left(\Omega / \mathbf{w}_{0}\right) .
$$

Similarly, the free energy $F_{d}(0)$ in eq. (28) can be exactly evaluated as

$$
F_{d}(0)= \begin{cases}\frac{\hbar}{2 \pi}\left\{(\Omega+\gamma) \ln \left(\frac{\Omega+\gamma}{\Omega}\right)+\gamma \ln \left(\frac{\Omega}{\mathbf{w}_{0}}\right)+2 \mathbf{w}_{1} \arccos \left(\frac{\gamma}{2 \mathbf{w}_{0}}\right)\right\} & \text { for } \quad \mathbf{w}_{0}>\gamma / 2 \\ \frac{\hbar}{2 \pi}\left\{(\Omega+\gamma) \ln \left(\frac{\Omega+\gamma}{\Omega}\right)+\gamma \ln \left(\frac{\Omega}{\mathbf{w}_{0}}\right)+\overline{\mathbf{w}}_{1} \ln \left(\frac{\gamma / 2-\overline{\mathbf{w}}_{1}}{\gamma / 2+\overline{\mathbf{w}}_{1}}\right)\right\} & \text { for } \quad \mathbf{w}_{0} \leq \gamma / 2 .\end{cases}
$$

Clearly, $\left(E_{s}\right)_{d}(0)$ and $F_{d}(0)$ for the underdamped case are identical to eqs. (10) and (14) in [11], respectively. These expressions can also be applied for the overdamped case $\left(\mathbf{w}_{1} \notin \mathbb{R}\right)$ with the aid of the complex-valued expression, $\arccos (y)=\frac{\pi}{2}+i \ln \left(i y+\sqrt{1-y^{2}}\right)$ and actually equivalent to those for the overdamped case derived here. Then, we can obtain $E_{s}(0)<F(0)$ for both cases (see Fig. 2 in [11]).

In the limit $\Omega \rightarrow \infty$ (equivalently, $\omega_{d} \rightarrow \infty$ ), we get from eqs. (65)-(68)

$$
K_{d} \approx \frac{\hbar \Omega}{2 \pi} \ln \left(\frac{\Omega+\gamma}{\Omega}\right) \approx \frac{\hbar \gamma}{2 \pi}\left(1-\frac{\gamma}{2 \Omega}\right)+\mathcal{O}\left(\frac{1}{\Omega^{2}}\right)
$$

which reduces to

$$
\frac{\gamma}{\pi \mathbf{w}_{0}} E_{g}=\frac{\hbar \gamma}{2 \pi} \frac{1}{\sqrt{1+\gamma / \Omega}} \approx \frac{\hbar \gamma}{2 \pi}\left(1-\frac{\gamma}{2 \Omega}\right)+\mathcal{O}\left(\frac{1}{\Omega^{2}}\right)
$$

(cf. eq. (15) in [11]). Lastly, we explicitly give an explicit expression for $K_{d}$ in original parameters $\left(\omega_{0}, \omega_{d}, \gamma_{o}\right)$,

$$
K_{d}=\frac{\hbar \gamma_{o}}{2 \pi} \int_{0}^{\infty} d \lambda \frac{2 \lambda_{d}^{2} \lambda^{5}-\left(2 \lambda_{0}^{2}+\lambda_{d}\right) \lambda_{d}^{2} \lambda^{3}}{\left\{\left(\lambda^{2}+\lambda_{d}^{2}\right)\left(\lambda^{2}-\lambda_{0}^{2}\right)-\lambda_{d} \lambda^{2}\right\}^{2}+\left(\lambda_{d}^{2} \lambda\right)^{2}},
$$

where a dimensionless parameter $\lambda=\omega / \gamma_{o}$ with $\lambda_{0}=\omega_{0} / \gamma_{o}$ and $\lambda_{d}=\omega_{d} / \gamma_{o}$. Here, we see that the non-zero value of $K_{d}$ for $\lambda_{d} \rightarrow \infty$ arises from the competition between two terms of the numerator of the integrand for large $\lambda$. Eq. (71) is also used in Table 2 for comparison with $K_{d, 1}$ in eq. (49).

\section{C : Details for eqs. (41) and (47)}

In derivation of eq. (41), we used the relationship 24] $E_{1}\left(-y \pm i 0^{+}\right)=-\operatorname{Ei}(y) \mp i \pi$, where the exponential integrals $E_{1}(y)=\int_{1}^{\infty} d z e^{-y z} / z$ and $\operatorname{Ei}(y)=P \int_{-\infty}^{y} d z e^{z} / z$. Also, for eq. (47), we employed, first, [25]

$$
\int_{0}^{\infty} d y \frac{\cos (a y)}{y+b}=-\sin (a b) \operatorname{si}(a b)-\cos (a b) \operatorname{Ci}(a b),
$$

where the sine integral $\operatorname{si}(y)=-\int_{y}^{\infty} d z \frac{\sin (z)}{z}=-\frac{\pi}{2}+\operatorname{Si}(y)$ with $\operatorname{Si}(y)=\int_{0}^{y} d z \frac{\sin (z)}{z}$, and the cosine integral $\operatorname{Ci}(y)=$ $-\int_{y}^{\infty} d z \frac{\cos (z)}{z}=c_{e}+\ln y+\int_{0}^{y} d z \frac{\cos (z)-1}{z}$ with the Euler constant $c_{e}=0.5772156649 \cdots ;$ secondly, we used the relations $\operatorname{Si}(i y)=\frac{i}{2}\left\{\operatorname{Ei}(y)+E_{1}(y)\right\}$ and $\operatorname{Ci}(i y)=\frac{1}{2}\left\{\operatorname{Ei}(y)-E_{1}(y)\right\}+\frac{\pi}{2} i[24]$. 


\section{References}

1. H.B. Callen, Thermodynamics and an introduction to thermostatics (2nd ed., John Wiley, 1985).

2. V. Špička, Th.M. Nieuwenhuizen, and P.D. Keefe, Physica E 29, 1 (2005) and references therein; D.P. Sheehan, ed., Quantum limits to the second law (AIP Conference Proceedings, No. 643, 2002); see also http://www.ipmt-hpm.ac.ru/SecondLaw/, Quantum limits to the second law of thermodynamics, Open Internet Conference and Information Center.

3. M.D. LaHaye, O. Buu, B. Camarota, and K.C. Schwab, Science 304, 74 (2004).

4. A.A.Clerk, Phys. Rev. B 70, 245306 (2004).

5. A. MacKinnon and A.D. Armour, Physica E 18, 235 (2003).

6. P. Hänggi and G.-L. Ingold, Chaos, 15, 026105 (2005).

7. J. Gemmer, M. Michel, and G. Mahler, Quantum Thermodynamics (Springer, Berlin, 2004).

8. X.L. Li, G.W. Ford, and R.F. O'Connell, Phys. Rev. E 51, 5169 (1995); K.E. Nagaev and M. Büttiker, Europhys. Lett., $\mathbf{5 8}(4), 475$ (2002).

9. G.W. Ford and R.F. O'Connell, Physica E 29, 82 (2005).

10. P. Hänggi and G.-L. Ingold, Acta Physica Polonica B 37, 1537 (2006).

11. G.W. Ford and R.F. O'Connell, Phys. Rev. Lett. 96, 020402 (2006).

12. G.-L. Ingold, Dissipative quantum systems in Quantum transport and dissipation (Wiley-VCH, 1998), pp 213-248.

13. Following, e.g., Refs. [6], [10], [12], and [15], we adopt the Laplace transforms for an analysis of the frequency domain in this paper, instead of the Fourier transforms which have been employed in a series of papers by Ford and O'Connell, e.g., Refs. [11, [9], and [22]. Clearly, both are a priori equally acceptable to give rise to the same desired physical results in the end.

14. G.E. Roberts and H. Kaufman, Table of Laplace Transforms (W.B. Saunders, Philadelphia, 1966).

15. A.M. Levine, M. Shapiro, and E. Pollak, J. Chem. Phys. 88, 1959 (1988).

16. G.W. Ford, J.T. Lewis, and R.F. O'Connell, J. Stat. Phys. 53, 439 (1988).

17. U. Weiss, Quantum dissipative systems (2nd ed., World Scientific, Singapore, 1999).

18. M. Büttiker and A.N. Jordan, Physica E 29, 272 (2005).

19. J. Oppenheim, M. Horodecki, P. Horodecki, and R. Horodecki, Phys. Rev. Lett. 89, 180402 (2002).

20. N.G. van Kampen, J. Stat. Phys. 115, 1057 (2004).

21. G. Alber, T. Beth, M. Horodecki, et al., Quantum Information: An introduction to basic theoretical concepts and experiments (Sprnger, Berlin, 2001).

22. G.W. Ford, J.T. Lewis, and R.F. O'Connell, Phys. Rev. Lett. 55, 2273 (1985).

23. J. Meixner, in Statistical mechanics of equilibrium and non-equilibrium, edited by J. Meixner (North-Holland, Amsterdam, 1965), pp 52-68.

24. M. Abramowitz and I. Stegun, Handbook of Mathematical Functions with Formulas, Graphs, and Mathematical Tables (Dover, New York, 1974).

25. I.S. Gradshteyn and I.M. Ryzhik, Table of Integrals, Series, and Products (6th ed., Academic Press, San Diego, 2000).

Fig. 11 $K_{d, 2} / E_{g}$ versus $x=\gamma / \mathbf{w}_{0}$, where the ground state energy $E_{g}=\frac{\hbar}{2} \sqrt{\Omega \gamma+\mathbf{w}_{0}^{2}}$; for $\Omega=2 \mathbf{w}_{0}$ (dot), $5 \mathbf{w}_{0}$ (solid), $10 \mathbf{w}_{0}$ (dashed) from top to bottom. 